\title{
Response of serum intact parathyroid hormone and milk calcium concentrations to vitamin $D$ supplementation in breast-feeding mothers
}

\author{
A. J. Lucey ${ }^{1}$, J. Y. Zhang ${ }^{1}$, L. Carrabine ${ }^{1}$, L. Nolan ${ }^{2}$, J. R. Higgins ${ }^{2}$, K. D. Cashman ${ }^{1}$ and M. Kiely ${ }^{1}$ \\ ${ }^{1}$ Vitamin D Research Group, School of Food and Nutritional Sciences, University College Cork and ${ }^{2}$ Anu Research Centre, \\ Department of Obstetrics and Gynaecology, Cork University Maternity Hospital, University College Cork, Ireland
}

Vitamin D status can influence serum intact parathyroid hormone (iPTH). While the Ca content of expressed breast milk (EBM) appears independent of maternal serum 25-hydroxyvitamin D (s25(OH)D) concentrations in mothers accustomed to both low ${ }^{(1)}$ and high $\mathrm{Ca}^{(2)}$ intakes, recent data in lactating PTH knockout mice suggest that iPTH may contribute to regulating milk Ca content and that this may be further influenced by maternal Ca intake ${ }^{(3)}$. Data on associations between circulating iPTH, s25(OH)D and milk Ca content are limited in human subjects.

We investigated whether increasing maternal s25(OH)D concentrations altered circulating iPTH and the Ca content of EBM by means of a double-blind randomised placebo-controlled trial in 100 lactating women across three intervention groups that received $20 \mu \mathrm{g} / \mathrm{d}$ of vitamin $\mathrm{D}_{3}$ (to achieve a total vitamin $\mathrm{D}$ intake of $\sim 25 \mu \mathrm{g} / \mathrm{d}$ ), $\pm 500 \mathrm{mg} \mathrm{Ca}$, or placebo over 12 weeks. Fasting serum iPTH and s25(OH)D were measured at baseline (BL) and endpoint (EP) using ELISA. Ca levels were quantified in EBM at four time points using atomic absorption spectrophotometry.

\begin{tabular}{|c|c|c|c|c|c|c|c|c|c|c|}
\hline & \multicolumn{3}{|c|}{ Placebo (n 27) } & \multicolumn{3}{|c|}{ Vitamin D3 $(20 \mu \mathrm{g} / \mathrm{d})(\mathrm{n} \mathrm{36})$} & \multicolumn{3}{|c|}{ Vitamin D3 $(20 \mu \mathrm{g} / \mathrm{d})+\mathrm{Ca}(500 \mathrm{mg} / \mathrm{d})(\mathrm{n} \mathrm{37})$} & \multirow[b]{2}{*}{$P$} \\
\hline & $\overline{\text { Median }}$ & $25^{\text {th }}$ & $75^{\mathrm{th}}$ & Median & $25^{\text {th }}$ & $75^{\text {th }}$ & Median & $25^{\text {th }}$ & $75^{\text {th }}$ & \\
\hline $\begin{array}{l}\mathbf{s 2 5}(\mathbf{O H}) \mathbf{D}(\mathbf{n m o l} / \mathbf{L}) \\
\mathrm{BL}^{1} \\
\mathrm{EP}\end{array}$ & $\begin{array}{l}45.16 \\
48.25^{\mathrm{a}}\end{array}$ & $\begin{array}{l}29.2 \\
36.7\end{array}$ & $\begin{array}{l}64.9 \\
65.6\end{array}$ & $\begin{array}{l}45.41 \\
78.41^{\mathrm{b}}\end{array}$ & $\begin{array}{l}38.1 \\
67.9\end{array}$ & $\begin{array}{l}54.2 \\
94.1\end{array}$ & $\begin{array}{l}39.46 \\
75.92^{b}\end{array}$ & $\begin{array}{l}30.1 \\
67.7\end{array}$ & $\begin{array}{l}62.6 \\
97.9\end{array}$ & $<0.0012$ \\
\hline $\begin{array}{l}\text { Serum iPTH }(\mathbf{p g} / \mathbf{m l}) \\
\mathrm{BL}^{1} \\
\text { EP }\end{array}$ & $\begin{array}{l}40.77 \\
46.57\end{array}$ & $\begin{array}{l}24.2 \\
27.1\end{array}$ & $\begin{array}{l}82.1 \\
67.9\end{array}$ & $\begin{array}{l}46.50 \\
37.79\end{array}$ & $\begin{array}{l}25.1 \\
25.2\end{array}$ & $\begin{array}{l}61.9 \\
49.1\end{array}$ & $\begin{array}{l}37.06 \\
41.37\end{array}$ & $\begin{array}{l}18.6 \\
24.0\end{array}$ & $\begin{array}{l}53.9 \\
63.1\end{array}$ & $0.345^{3}$ \\
\hline $\begin{array}{l}\text { Ca (mg/100 ml milk) } \\
\text { BL (Wk 0) } \\
W^{4} \\
\text { Wk } 8 \\
\text { EP (Wk 12) }\end{array}$ & $\begin{array}{l}31.13 \\
30.21 \\
30.67 \\
32.53\end{array}$ & $\begin{array}{l}26.3 \\
26.5 \\
26.2 \\
26.1\end{array}$ & $\begin{array}{l}35.1 \\
34.8 \\
38.9 \\
35.9\end{array}$ & $\begin{array}{l}27.41 \\
28.84 \\
29.36 \\
28.32\end{array}$ & $\begin{array}{l}24.7 \\
25.4 \\
26.5 \\
24.7\end{array}$ & $\begin{array}{l}30.7 \\
34.2 \\
34.6 \\
34.2\end{array}$ & $\begin{array}{l}29.17 \\
31.06 \\
29.14 \\
30.92\end{array}$ & $\begin{array}{l}25.4 \\
25.1 \\
25.5 \\
23.8\end{array}$ & $\begin{array}{l}38.5 \\
37.0 \\
36.8 \\
36.2\end{array}$ & 0.6054 \\
\hline
\end{tabular}

Data are median, 25th and 75th percentile. ${ }^{1}$ ANOVA BL, all $P>0.1 .{ }^{2}$ ANCOVA adjusting for BL s25(OH)D, vitamin D intake and season of EP blood sampling; ${ }^{3}$ ANCOVA adjusting for BL s25(OH)D, iPTH, age, parity and \% body fat. ${ }^{4}$ One-way repeated measures ANOVA adjusting for BL s25(OH)D, iPTH, \% body fat and parity.

At BL, the median vitamin D and Ca intakes were $5.9 \mu \mathrm{g}$ and $1243 \mathrm{mg} / \mathrm{d}$ and $25 \%$ were below the Ca EAR of $800 \mathrm{mg} / \mathrm{d}^{(4)}$. Ca and vitamin D intakes were not associated with serum iPTH levels or the Ca content of EBM $(P>0.1)$. Serum iPTH at BL was associated with s25(OH)D $(\beta-0.327, P<0.001), \%$ body fat $(\beta 0.330, P<0.001)$, previous pregnancy $(\beta 0.286, P=0.004)$ and maternal age $(\beta 0.167$, $P=0.08)\left(\right.$ adj. $R^{2}$ 0.293). EBM Ca concentrations at BL were associated with iPTH $(\beta-0.327, P=0.01)$ and $\%$ body fat $(\beta 0.272$, $P=0.02)$ but not with s25(OH)D concentrations $(\beta-0.187, P=0.1)\left(\right.$ adj. $\left.R^{2} 0.066\right)$. The intervention substantially increased s25(OH)D levels in the treatment groups $(P<0.001)$ and reduced the $\%$ of women with s $25(\mathrm{OH}) \mathrm{D}$ levels $<50 \mathrm{nmol} / 1$ from 63 to $4 \%$, but had no significant effect on circulating iPTH or EBM Ca concentrations $(P>0.1)$.

In conclusion, while vitamin D supplementation at levels sufficient to achieve an s25(OH)D level of $50 \mathrm{nmol} / 1$ in most women did not significantly influence circulating iPTH concentrations or the Ca content of EBM, these data show associations between iPTH, adiposity and the $\mathrm{Ca}$ content of human milk, which warrant further investigation.

Funded by the Irish Government Department of Agriculture, Fisheries and Food through the Food Institutional Research Measure.

1. Prentice A, Yan LMA, Jarjou B et al. (1997) Acta Paediatr 86, 1006-1008.

2. Basile LA, Taylor SN, Wagner CL et al. (2006) Breastfeed Med 1, 27-35.

3. Cao G, Gu Z, Ren Y et al. (2009) Endocrinology 150, 561-569.

4. IOM (2010) Dietary Reference Intakes for Calcium and Vitamin D. http://www.iom.edu/vitamind 